\title{
étude des matériaux marneux utilisés pour la construction du barrage de Montbel (09)
}

\author{
study of the marly materials used in the construction \\ of the Montbel dam (09)
}

\author{
E. ALONSO \\ Ingénieur, C.E.M.A.G.R.E.F. Bordeaux* \\ D. LOUDIÈRE \\ I.C.G.R.E.F., C.E.M.A.G.R.E.F. Antony* * \\ P. MORLIER \\ Professeur, Université Bordeaux I***
}

\section{Résumé}

La nature des marnes rend leur utilisation très difficile pour la construction de remblais et de barrages. Dans cette étude les marnes évolutives du site de Montbel sont étudiées en fonction de leur degré d'altération. Les essais effectués montrent que ces marnes doivent être compactées jusqu'à une densité sèche comprise entre le maximum du Proctor Normal et la densité sèche en place. En outre il est constaté un comportement mécanique différent suivant que le compactage du matériau ait lieu sur le chantier à l'aide d'un rouleau à pieds dameurs ou en laboratoire.

\footnotetext{
Abstract

The nature of marls makes them very difficult to use in the construction of fills and dams. In this study the evolutive marls on the Montbel site are studied with respect to the degree of weathering they undergo. The tests carried out demonstrate that these marls must be compacted until they have a dry density comprised between the maximum Proctor and dry in situ densities. In addition, a different behaviour was noted depending upon whether the material was compacted on site with a tamping roller, or in the laboratory.
} 


\section{INTRODUCTION}

Le projet de construction, dans le département de l'Ariège, du barrage de Montbel, dans un site où l'utilisation de la marne s'est imposée pour la réalisation du barrage principal et des autres ouvrages, nous a conduits à entreprendre de nombreuses études et de nombreux essais.

En outre, on a pu constater que ce matériau particulier avait posé des problèmes importants lors des études et des travaux concernant d'autres ouvrages (barrages et remblais) : les incidents survenus montrent bien qu'il s'agit d'un matériau difficile.

En effet, non seulement la marne est un matériau intermédiaire entre les argiles et les calcaires, entre les sols et les roches, mais surtout des marnes peuvent évoluer, en présence d'eau, plus ou moins rapidement, d'un aspect rocheux à celui d'une boue.

Comme la marne, relativement fréquente en France, surtout dans le Bassin Parisien, le Sud-Ouest et le SudEst, est de plus en plus utilisée pour la construction d'ouvrages, il nous a paru intéressant, notamment pour les projeteurs confrontés à ce type de matériau, de présenter les différents résultats obtenus; après un chapitre consacré aux marnes en général, les différentes études des matériaux marneux du site de Montbel sont traitées.

\section{LES MARNES}

En ce qui concerne la définition des marnes, FILLIAT et DEPREZ (1) considèrent qu'elles sont le terme moyen d'une série continue qui va du calcaire à l'argile, selon le tableau I (d'après CORRENS) :

On peut ajouter la définition de HERAUD, RESTITUITO et LE ROUX (2) : * la dénomination de marne désigne une roche intermédiaire entre l'argile et le calcaire; le pourcentage de $\mathrm{CaCO}_{3}$ n'est pas le seul critère de détermination, il s'y ajoute une notion de comportement ou de sensibilité à l'eau qui fait d'une marne un matériau évolutif. Cette notion restrictive donne des marnes une mauvaise image de marque, mais c'est celle qu'elles ont dans la pratique».
Comme ce texte est consacré aux marnes en tant que matériaux susceptibles d'être utilisés pour la construction d'un barrage, il faut, à notre avis, prendre en considération les trois aspects suivants :

- lorsqu'il s'agit de trouver des matériaux pour la construction d'un ouvrage, on s'intéresse notamment aux couches superficielles, et donc les phénomènes d'altération naturelle interviennent : ainsi, comme avec la plupart des roches, on peut avoir en surface une couche altérée meuble (aspect d'un sol) et à quelques mètres de profondeur, une marne dure (aspect d'une roche);

- en second lieu, on appelle marne des matériaux qui vont d'une roche dure, dont la résistance à la compression simple peut dépasser une dizaine de $\mathrm{MPa}$, à une argile peu carbonatée et peu consolidée d'une résistance à la compression simple de quelques dizièmes de $\mathrm{MPa}$, matériau finalement proche d'un sol dans ce dernier cas;

- troisièmement, les marnes plus ou moins dures qui à l'extraction sont sous forme de blocs et de cailloux, peuvent par la suite se déliter; par exemple un échantillon de marne dure de la dimension d'un caillou mis dans un récipient contenant de l'eau peut se transformer en boue en quelques heures, voire en quelques minutes; bien entendu, cette désagrégation brutale dépend de la composition et de la texture du matériau.

En ce qui concerne la composition minéralogique des marnes on rencontre peu souvent un mélange d'argiles et de calcaire uniquement. En général les marnes sont composées des trois phases suivantes : argiles, carbonate de calcium $\left(\mathrm{CaCO}_{3}\right)$, quartz (silt, sable); d'autres éléments peuvent également être présents : feldspaths, micas, micro-organismes, dolomite, gypse, pyrite, etc.

Si le pourcentage d'argile et la nature des minéraux argileux ont une influence sur le comportement de la marne, la texture joue également un rôle important.

La texture concerne l'arrangement spatial, la forme et la dimension des minéraux d'une roche ou d'un sol; elle peut être étudiée à l'aide du microscope électronique à balayage. Des grossissements de 1000 à 10000 sont en général effectués pour l'étude de la texture des

Tableau I.

\begin{tabular}{|c|c|c|c|}
\hline$\% \mathrm{CaCO}_{3}$ & $\%$ argiles & Nom de la roche & Comportement \\
\hline \multirow{3}{*}{10} & \multirow[t]{2}{*}{100} & & \multirow[b]{2}{*}{ Plastique } \\
\hline & & Argile & \\
\hline & SF & Marne argileuse & \multirow{2}{*}{$\begin{array}{l}\text { Plus ou moins } \\
\text { raide }\end{array}$} \\
\hline 35 & \multirow{2}{*}{35} & Marne & \\
\hline 65 & & Calcaire marneux & \multirow{2}{*}{ Rigide } \\
\hline $\begin{array}{r}90 \\
100\end{array}$ & $\begin{array}{r}10 \\
0\end{array}$ & Calcaire & \\
\hline
\end{tabular}


marnes. On peut ainsi apprécier notamment la disposition des différents minéraux les uns par rapport aux autres (par exemple particules d'argile enrobant les carbonates), leurs liaisons (par exemple grains de $\mathrm{CaCO}_{3}$ soudés entre eux), la forme et la taille des différents constituants, la porosité du matériau, sa fissuration. On peut observer des textures homogènes (compactes ou non), des textures orientées (direction privilégiée en particulier des minéraux argileux), des textures en microagrégats; il s'agit dans ce dernier cas, de matériaux composés d'agrégats d'une taille de quelques microns à quelques centaines de microns, ces agrégats étant constitués de plusieurs petites particules (argile, calcite, quartz...); on a donc une porosité interagrégats et une porosité intra-agrégats.

En ce qui concerne les liaisons calcaires, leur rôle peut être très important; lors de la diagenèse des marnes, des liaisons entre grains de $\mathrm{CaCO}_{3}$ peuvent se former; elles sont alors de nature ionique, comme celles qui existent à l'intérieur des grains. Si elles sont suffisamment nombreuses et fortes, elles s'opposent en présence d'eau à l'action de l'argile et ainsi, le matériau marneux a un comportement de roche; dans le cas contraire, le gonflement de l'argile fait passer la marne de P'état de roche à celui de sol.

Pour mieux apprécier le caractère évolutif des marnes, LEROUX (3) propose l'utilisation d'un simulateur d'altération constitué d'un émetteur infra-rouge et ultraviolet, d'une soufflerie d'air chaud et d'un bac d'immer- sion contenant de l'eau; il s'agit de répéter des cycles humidification-dessication avec cet appareil. On peut ainsi constater l'aptitude des marnes à évoluer d'une roche vers un sol et leur vitesse d'évolution. En général, les marnes qui ont de faibles teneurs en carbonates (10 à $20 \%$ ) associées à une phase argileuse importante (au moins $30 \%$ ) ont une évolution très rapide. Par contre, les marnes qui contiennent suffisamment de grains de $\mathrm{CaCO}_{3}$ bien soudés entre eux, n'évoluent pratiquement pas. Les marnes à texture homogène et compacte ont en général une évolution lente, tandis que les marnes à texture en microagrégats se désagrègent rapidement. L'altération en place peut également jouer un rôle et entraîner une évolution plus rapide des matériaux prélevés plus près de la surface.

Il faut bien distinguer d'une part l'altération en place d'une marne plus ou moins décomprimée et fissurée, qui est un phénomène lent et progressif se traduisant notamment en surface par la présence d'un sol décarbonaté qui peut être relativement compact (dissolution de $\mathrm{CaCO}_{3}$ par l'eau chargée de $\mathrm{CO}_{2}$ ); d'autre part il y a l'altération à laquelle est soumise une marne évolutive (plus ou moins dure et sèche), extraite à une certaine profondeur, lorsqu'on la met en présence d'eau, ce qui entraîne un gonflement de l'argile et une désagrégation du matériau, qui peut être très rapide, aucune dissolution n'intervenant.

Finalement et schématiquement, on peut établir la classification suivante des marnes :

Tableau II.

\begin{tabular}{|c|c|c|c|c|c|c|}
\hline \multirow{3}{*}{$\begin{array}{c}\text { Nature } \\
\text { de la marne } \\
\text { en place }\end{array}$} & \multirow{3}{*}{$\begin{array}{c}\text { Marne altérée } \\
\text { meuble } \\
\text { (sol) }\end{array}$} & \multirow{2}{*}{\multicolumn{2}{|c|}{$\begin{array}{l}\text { Marne intermédiaire entre sol } \\
\text { et roche }\end{array}$}} & \multicolumn{3}{|c|}{ Marne à aspect rocheux, dure et sèche } \\
\hline & & & & \multicolumn{2}{|c|}{ Pas ou peu de liaisons $\mathrm{CaCO}_{3}$} & \multirow[b]{2}{*}{$\begin{array}{l}\text { Liaisons } \\
\text { calcaires } \\
\text { abondantes } \\
\text { et solides }\end{array}$} \\
\hline & & $\begin{array}{l}\text { Matériau } \\
\text { partiel- } \\
\text { lement } \\
\text { altéré }\end{array}$ & $\begin{array}{l}\text { Matériau } \\
\text { ni très } \\
\text { consolidé } \\
\text { ni très } \\
\text { carbonaté }\end{array}$ & $\begin{array}{c}\text { Texture } \\
\text { en } \\
\text { microagrégats }\end{array}$ & $\begin{array}{c}\text { Texture } \\
\text { homogène, } \\
\text { compacte }\end{array}$ & \\
\hline $\begin{array}{l}\text { Caractéris- } \\
\text { tiques } \\
\text { du matériau } \\
\text { en place }\end{array}$ & $\begin{array}{l}\text { Matériau } \\
\text { humide, } \\
\text { décarbonaté } \\
\text { en général } \\
\text { peu profond }\end{array}$ & $\begin{array}{c}\text { Matériau } \\
\text { moins sec } \\
\text { et moins } \\
\text { compact que } \\
\text { le matériau } \\
\text { intact }\end{array}$ & $\begin{array}{l}\text { Matériau } \\
\text { argileux } \\
\text { plutôt } \\
\text { tendre }\end{array}$ & $\begin{array}{l}\text { Vides plus } \\
\text { ou moins } \\
\text { importants } \\
\text { entre } \\
\text { les agrégats } \\
\text { et à l'intérieur } \\
\text { de } \\
\text { ces derniers }\end{array}$ & $\begin{array}{l}\text { Peu de vides } \\
\text { à l'intérieur } \\
\text { du matériau }\end{array}$ & $\begin{array}{l}\text { En général } \\
\text { matériau } \\
\text { contenant } \\
\text { plus de } 50 \% \\
\text { de } \mathrm{CaCO}_{3}\end{array}$ \\
\hline \multirow[t]{2}{*}{$\begin{array}{c}\text { Compor- } \\
\text { tement } \\
\text { en présence } \\
\text { d'eau } \\
\text { du matériau } \\
\text { prélevé }\end{array}$} & \multirow[t]{2}{*}{$\begin{array}{c}\text { Compor- } \\
\text { tement } \\
\text { analogue aux } \\
\text { sols argileux }\end{array}$} & $\begin{array}{l}\text { Se désagrège } \\
\text { plus vite que } \\
\text { les matériaux } \\
\text { moins altérés }\end{array}$ & $\begin{array}{l}\text { Désagréga- } \\
\text { tion d'autant } \\
\text { plus rapide } \\
\text { que le } \\
\text { matériau est } \\
\text { plus argileux }\end{array}$ & $\begin{array}{l}\text { Ce matériau } \\
\text { se désagrège } \\
\text { rapidement }\end{array}$ & $\begin{array}{c}\text { Ce matériau } \\
\text { se désagrège } \\
\text { lentement }\end{array}$ & \multirow[t]{2}{*}{$\begin{array}{l}\text { Matériau } \\
\text { pratiquement } \\
\text { insensible } \\
\text { à l'eau. } \\
\text { Se comporte } \\
\text { comme } \\
\text { une roche }\end{array}$} \\
\hline & & \multicolumn{4}{|c|}{$\begin{array}{l}\text { L'ampleur de la désagrégation dépend notamment } \\
\text { des vides disponibles autour du matériau }\end{array}$} & \\
\hline
\end{tabular}




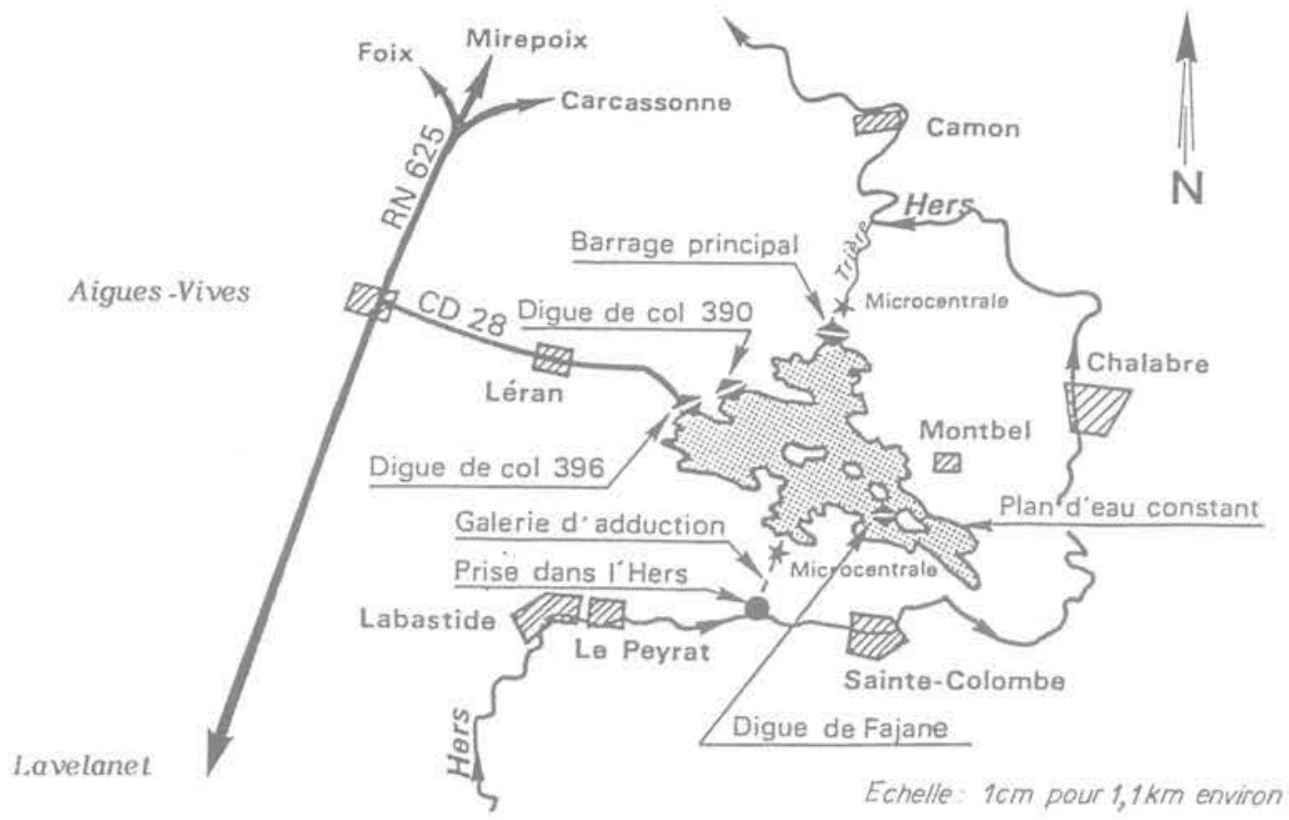

Fig. 1. - Plan de situation.

\section{AMÉNAGEMENT DE MONTBEL ET TRAVAUX DE RECONNAISSANCE}

\subsection{L'aménagement de Montbel}

Afin de permettre le développement des irrigations dans les départements de l'Ariège, de l'Aude et de la Haute-Garonne, a été décidée la création d'un réservoir d'une capacité de 60 millions de $\mathrm{m}^{3}$, à Montbel. $\mathrm{Ce}$ volume est obtenu en noyant 550 hectares de la cuvette naturelle de Montbel dont la fermeture est assurée par un barrage d'une hauteur maximum de $36 \mathrm{~m}$ (sur le ruisseau de la Trière, affluent rive gauche de l'Hers) et par deux digues de col (hauteurs maximales de $13 \mathrm{~m}$ et de $7 \mathrm{~m}$ )

L'alimentation de la retenue (dont le bassin versant propre est seulement de $14,5 \mathrm{~km}^{2}$ ) est réalisée à l'aide d'une galerie d'adduction permettant la dérivation d'un débit de $10 \mathrm{~m}^{3} / \mathrm{s}$ des eaux excédentaires d'hiver et de printemps de l'Hers (affluent rive droite de l'Ariège).

L'ensemble des ouvrages se trouve sur le plan ci-dessous (fig. 1) ; la digue de Fajane, d'une hauteur maximale de $20 \mathrm{~m}$, a pour but de maintenir un plan d'eau constant à vocation touristique.

Tous les remblais ont été exécutés à l'aide des matériaux marneux disponibles dans la zone noyée. Le barrage principal (volume d'environ $800000 \mathrm{~m}^{3}$ ) a été réalisé en 1983 et la digue de Fajane (volume d'environ $300000 \mathrm{~m}^{3}$ ) en 1984. Le remplissage de la retenue était presque terminé au début de l'été 1985 (fig. 2).
Les principaux organismes concernés par le réservoir de Montbel sont les suivants :

- Ministère de l'Agriculture présentant le dossier et assurant la majorité du financement de l'opération (coût total d'environ 180 millions de francs H.T.);

- Institution Interdépartementale pour l'Aménagement du barrage de Montbel : Maître d'ouvrage;

- Direction Départementale de l'Agriculture de l'Ariège: conducteur d'opération (maître d'cuvre pour la digue de Fajane);

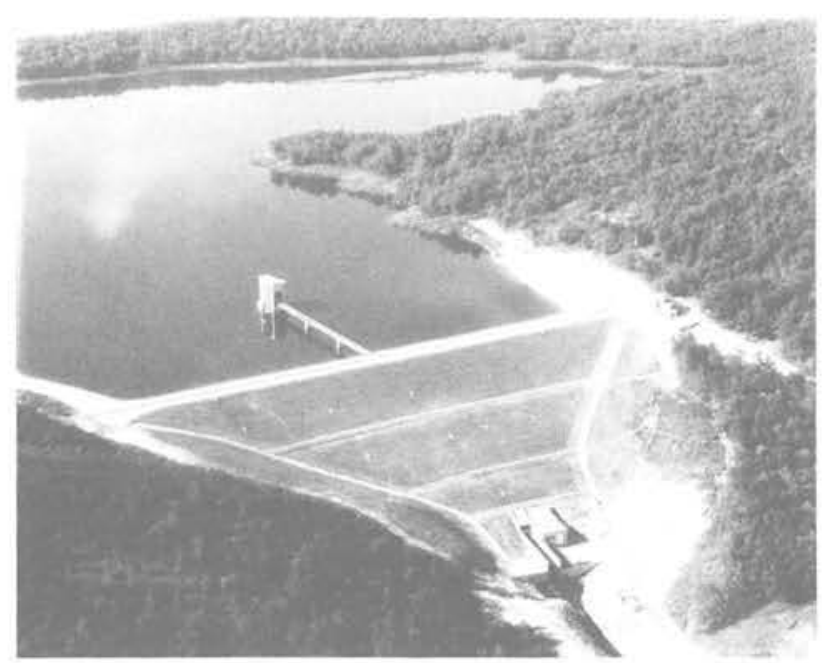

Fig. 2. - Vue du barrage principal. 


\section{- Bureau d'Ingénieurs Conseils COYNE et BELLIER : Maître d'œuvre;}

- Entreprise Grands Travaux de Marseille : exécutant principal (Entreprise BEC, pour la digue de Fajane).

Les études géologiques et géotechniques, commencées en 1978, ont été effectuées par le Centre National du Machinisme Agricole, du Génie Rural, des Eaux et des Forêts (C.E.M.A.G.R.E.F.) - Groupement de Bordeaux (4), avec la participation des géologues de la Compagnie Nationale d'Aménagement de la Région du Bas-Rhône et du Languedoc.

\subsection{Les travaux de reconnaissance}

Les couches qui affleurent dans la cuvette de Montbel appartiennent au Lutétien; essentiellement marneuses, elles ont pratiquement un pendage Sud-Nord de quelques degrés.

Lors de la recherche des matériaux susceptibles d'être utilisés pour la construction du barrage principal et des deux digues de col, les reconnaissances suivantes ont été effectuées dans la zone noyée :

- une reconnaissance des couches superficielles à l'aide d'une pelle hydraulique sur chenilles d'une puissance de $100 \mathrm{ch}$; cent cinq tranchées ont été exécutées,

- une reconnaissance de la butte de Taurine, située en rive droite à quelques centaines de mètres du barrage principal, à l'aide d'une sondeuse (carottes : diamètre $90 \mathrm{~mm}$ ); trois sondages ont été exécutés.

En fond de vallée (Trière), des dépôts d'alluvions récents constituent une couche limoneuse humide d'une épaisseur comprise entre $2,50 \mathrm{~m}$ et $5,50 \mathrm{~m}$; ces limons recouvrent une mince couche de grave ou reposent directement sur le substratum marneux. Ces limons, peu plastiques (limite de liquidité moyenne égale à $30 \%$ et limite de plasticité moyenne égale à $19,5 \%)$, ont une teneur en eau naturelle moyenne de
$21 \%$ (donc légèrement supérieure à $\omega_{p}$ ) quelle que soit l'époque du prélèvement et quel que soit la profondeur de ce dernier; la teneur en eau optimale des essais de compactage Proctor Normal est comprise entre $13 \%$ et $15 \%$ : le séchage de ces matériaux limoneux proches de la saturation s'avérant très difficile, il est alors décidé de réaliser le barrage à l'aide des matériaux marneux disponibles dans les versants.

Les tranchées exécutées dans les versants marneux ont une profondeur variant de $1,80 \mathrm{~m}$ à $4,50 \mathrm{~m}$. Sous la terre végétale, on trouve une marne meuble (éluvions, colluvions de versant), ensuite une marne très tendre, puis de moins en moins tendre à mesure que la profondeur augmente; finalement, on arrive en général sur une marne sèche et dure, le creusement à la pelle devenant très difficile.

Les marnes meubles sont des marnes complètement altérées qui ont l'aspect d'un sol argileux classique ; par contre, lors de l'extraction, les marnes sous-jacentes viennent essentiellement sous forme de blocs et de cailloux tendres à durs. Ces marnes sous-jacentes, partiellement altérées, sont classées en fonction de leur teneur en eau naturelle, qui dépend de leur profondeur et de leur plasticité :

- marnes tendres, dont la teneur en eau est supérieure à $10 \%$;

- marnes dures, dont la teneur en eau est inférieure à $10 \%$.

Si on considère l'ensemble des tranchées exécutées dans la marne, on obtient la coupe moyenne suivante :

- $0,40 \mathrm{~m}$ de terre végétale;

- $1 \mathrm{~m}$ de marnes complètement altérées meubles, dont la teneur en eau naturelle, variable en surface suivant les conditions atmosphériques, est comprise entre $13 \%$ et $23 \%$;

- $1,50 \mathrm{~m}$ de marnes partiellement altérées tendres, dont la teneur en eau naturelle, constante, est comprise entre $10 \%$ et $17 \%$;

- des marnes partiellement altérées dures dont la teneur en eau, constante, est comprise entre $6 \%$ et $10 \%$.

Tableau III. - Teneurs en eau moyennes suivant l'altération (en \%)

\begin{tabular}{|l|c|c|c|}
\hline \multicolumn{1}{|c|}{ Marne } & intacte & $\begin{array}{c}\text { partiellement } \\
\text { altérée }\end{array}$ & meuble \\
\hline $\begin{array}{l}\text { à forte plasticité (limite } \\
\text { de liquidité proche de 55) }\end{array}$ & $\omega=11$ & $\omega=16$ & $\omega=21$ \\
\hline $\begin{array}{l}\text { à moyenne plasticité (limite } \\
\text { de liquidité proche de 40) }\end{array}$ & $\omega=8$ & $\omega=11$ & $\omega=16$ \\
\hline $\begin{array}{l}\text { à faible plasticité (limite } \\
\text { de liquidité proche de 30) }\end{array}$ & $\omega=6$ & $\omega=8$ & $\omega=13$ \\
\hline
\end{tabular}


Les coupes varient de quelques décimètres de marne meuble directement sur de la marne dure (transition négligeable) à plus de quatre mètres de marne meuble et de marne tendre.

Comme l'exploitation des marnes superficielles jugées convenables, les marnes meubles et les marnes tendres, conduit à des zones d'emprunts très étendues, il est décidé d'étudier également les marnes profondes de la butte de Taurine.

Les trois sondages carottés réalisés, d'une longueur de $30 \mathrm{~m}, 20 \mathrm{~m}$ et $15 \mathrm{~m}$, permettent de constater que cette butte est constituée de marnes indurées et sèches. A partir d'une profondeur de $2 \mathrm{~m}$, tous les échantillons prélevés ont une teneur en eau comprise entre $4,5 \%$ et $12 \%$

Schématiquement, on peut suivre sur le tableau III les teneurs en eau moyennes d'une même marne suivant son degré d'altération.

\section{IDENTIFICATION ET ESSAIS DE COMPACTAGE}

\subsection{Identification des marnes de Montbel}

Les marnes de Montbel sont constituées essentiellement d'éléments fins. Presque toutes les particules ont un diamètre inférieur à $80 \mu$ et 15 à $50 \%$ des éléments ont un diamètre équivalent inférieur à $2 \mu$. La densité spécifique moyenne des particules est égale à 2,73 (valeurs mesurées entre 2,70 et 2,76 ). Il s'agit d'un matériau surconsolidé, peu carbonaté et évolutif : des cailloux de marne, tendres ou durs, provenant des couches superficielles ou des couches profondes, plongés dans de l'eau, mettent quelques heures à quelques jours pour se déliter complètement.

Les marnes meubles ont l'aspect d'un sol argileux. En place, ce matériau a une densité sèche voisine du maximum obtenu à l'essai de compactage Proctor Normal (PN)

Les marnes partiellement altérées tendres ( $\omega$ entre $10 \%$ et $17 \%$ ) ont l'aspect d'une roche plus ou moins tendre. En place, ce matériau a une densité séche comprise entre 1,8 et 2,1 ce qui correspond en moyenne à 1,10 fois l'optimum PN.

Les marnes partiellement altérées dures ( $\omega$ entre $6 \%$ et $10 \%$ ) ont l'aspect d'une roche assez dure. En place, ce matériau a une densité sèche comprise entre 2,1 et 2,3 ce qui correspond à environ 1,15 fois l'optimum PN.

Les marnes intactes (entre 2 et $30 \mathrm{~m}$ de profondeur dans la butte de Taurine) ont l'aspect d'une roche dure; les résistances à la compression simple varient de $1 \mathrm{MPa}$ à plus de $10 \mathrm{MPa}$. En place, ce matériau a une densité sèche comprise entre 2 et 2,4 ce qui correspond à environ 1,20 fois l'optimum PN.
Les marnes partiellement altérées et les marnes intactes (butte de Taurine) contiennent 10 à $20 \%$ de $\mathrm{CaCO}_{3}$ (moyenne de $15 \%$ ) et un degré de saturation de lordre de $95 \%$; par contre les marnes meubles contiennent en général très peu de carbonates (moyenne de $4 \%$ ) et leur degré de saturation est variable.

En ce qui concerne la composition minéralogique des marnes de Montbel il y a, outre les carbonates $\left(\mathrm{CaCO}_{3}\right)$, du quartz et des minéraux argileux : en gros $50 \%$ de kaolinite, $30 \%$ d'illite et $20 \%$ de smectite. Les valeurs de la capacité d'échange de base (C.E.B.). mesurée sur la fraction inférieure à $2 \mu$, sont proches de 20 milliéquivalents (pour $100 \mathrm{~g}$ de sol) ; elles traduisent une teneur en argile vraie peu importante (particules de quartz dans la fraction inférieure à $2 \mu$ ). Les analyses thermiques différentielles effectuées montrent que les marnes ne contiennent pas de matières organiques, ni de gels d'hydroxydes de fer.

La détermination de la texture des marnes de Montbel, à l'aide du microscope électronique à balayage, a été demandée à LE ROUX (Laboratoire Central des Ponts et Chaussées); la photo de la figure 3 montre qu'il s'agit d'une texture en microagrégats où les particules d'argile enrobent les grains de quartz et de $\mathrm{CaCO}_{3}$.

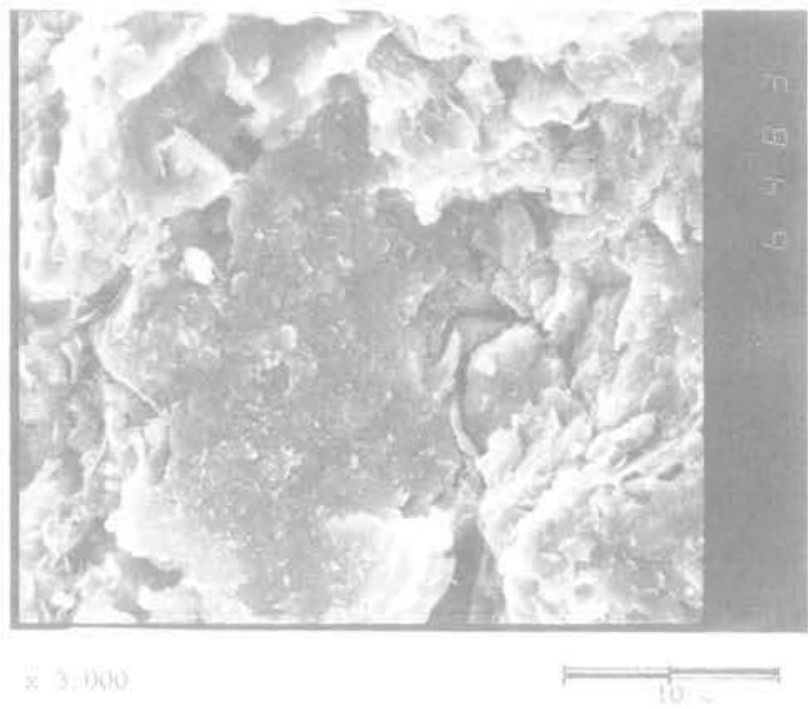

Fig. 3. - Texture de l'échantillon $\angle 2-2,10 \mathrm{~m}$ (marne partiellement altérée tendre). Les agrégats,

constitués d'une association argile quartz carbonate, ont une dimension de quelques dizaines de microns. Le contour de l'agrégat central est bien visible.

Les échantillons de marne partiellement altérée (prélevés sous forme de cailloux et de blocs) et les échantillons de marne intacte (prélevés sous forme de carottes), destinés aux analyses granulométriques et à la détermination des limites d'Atterberg, ont été brisés en morceaux et placés dans des récipients remplis d'eau pendant au moins 24 heures, afin qu'ils se délitent. 
Tableau IV. - Analyses granulométriques et limites d'Atterberg Valeurs moyennes

\begin{tabular}{|l|c|c|c|c|c|c|}
\hline \multirow{2}{*}{ Marnes } & \multicolumn{2}{|c|}{ meubles } & \multicolumn{2}{c|}{$\begin{array}{c}\text { partiellement } \\
\text { altérées }\end{array}$} & \multicolumn{2}{c|}{ intactes } \\
\cline { 2 - 7 } & $\omega_{L} \geqslant 40$ & $\omega_{L}<40$ & $\begin{array}{c}\omega \geqslant 10 \\
\text { tendres }\end{array}$ & $\begin{array}{c}\omega<10 \\
\text { dures }\end{array}$ & $\begin{array}{c}\omega \geqslant 7 \\
\text { argileuses }\end{array}$ & $\begin{array}{c}\omega<7 \\
\text { gréseuses }\end{array}$ \\
\hline Nombre d'essais & 12 & 6 & 25 & 15 & 8 & 11 \\
\hline Pourcentage $<2 \mu$ & 35 & 23 & 33 & 23 & 32 & 21 \\
\hline Limite de liquidité \% & 48 & 35,5 & 43 & 35 & 40 & 30 \\
\hline Indice de plasticité & 25,5 & 16,5 & 22,5 & 16 & 20,5 & 12,5 \\
\hline Activité IP/\% $\% 2 \mu$ & 0,73 & 0,72 & 0,68 & 0,70 & 0,64 & 0,60 \\
\hline
\end{tabular}

Dans le tableau IV, les résultats moyens obtenus sur les échantillons des différentes marnes soumis à des essais d'identification (analyses granulométriques et limites d'Atterberg), sont regroupés : on considère d'une part les marnes meubles dont la limite de liquidité est inférieure à $40 \%$ (ou IP $<20$ ) et d'autre part celles dont la limite de liquidité est supérieure ou égale à $40 \%$ $(\mathrm{IP} \geqslant 20)$; dans l'ensemble cette séparation correspond à une teneur en eau de $16 \%$. Les marnes intactes sont également classées en deux catégories : les marnes argileuses dont la teneur en eau naturelle est supérieure ou égale à $7 \%$ et les marnes gréseuses dont la teneur en eau est inférieure à $7 \%$ (la limite de liquidité de $33,5 \%$ sépare aussi ces deux marnes).

Il y a une bonne continuité entre les marnes meubles, les marnes partiellement altérées et les marnes intactes. L'activité des argiles, d'après SKEMPTON, dans l'ensemble d'ailleurs assez faible, diminue avec la plasticité, des marnes meubles aux marnes intactes. En ce qui concerne les marnes non meubles, il semble donc que lors de la détermination des limites d'Atterberg, des particules d'argile restent agrégées et ne jouent pas totalement leur rôle, ce phénomène étant plus important pour les marnes intactes que pour les marnes partiellement altérées.

La relation entre l'indice de plasticité IP et la limite de liquidité $\omega_{L}$ peut s'écrire : IP $=0,73\left(\omega_{L}-13 \%\right)$ ce qui correspond à une droite située au-dessus de la ligne A d'équation IP $=0,73\left(\omega_{L}-20 \%\right)$ dans l'abaque de plasticité de CASAGRANDE.

\subsection{Essais de compactage (laboratoire)}

La réalisation d'un essai de compactage Proctor Normal consiste tout d'abord à réduire $12 \mathrm{~kg}$ de marne en petits cailloux qui sont laissés à l'air libre pendant quelques jours afin que leur teneur en eau diminue. Ensuite, le matériau est écrasé en petits éléments inférieurs à $5 \mathrm{~mm}$. Puis le matériau, divisé en cinq échantillons, est humidifié de +2 à +10 points environ (par exemple le matériau séché ayant une teneur en eau de $7 \%$, les cinq échantillons ont respectivement $9 \%, 11 \%, 13 \%, 15 \%$ et $17 \%$ de teneur en eau).

Tableau V. - Essais de compactage Proctor Normal - Valeurs moyennes

\begin{tabular}{|l|c|c|c|c|c|c|}
\hline \multirow{2}{*}{ Marnes } & \multicolumn{2}{|c|}{ meubles } & \multicolumn{2}{c|}{$\begin{array}{c}\text { partiellement } \\
\text { altérées }\end{array}$} & \multicolumn{2}{c|}{ intactes } \\
\cline { 2 - 7 } & $\omega_{L} \geqslant 40$ & $\omega_{L}<40$ & $\begin{array}{c}\omega \geqslant 10 \\
\text { tendres }\end{array}$ & $\begin{array}{c}\omega<10 \\
\text { dures }\end{array}$ & $\begin{array}{c}\omega \geqslant 7 \\
\text { argileuses }\end{array}$ & $\begin{array}{c}\omega<7 \\
\text { gréseuses }\end{array}$ \\
\hline Nombre d'essais & 6 & 1 & 11 & 4 & 3 & 2 \\
\hline Pourcentage $<2 \mu$ & 31 & 15 & 29 & 25 & 27 & 21 \\
Indice de plasticité & 25 & 19 & 21,5 & 18,5 & 18 & 13 \\
\hline Teneur en eau naturelle (\%) & 18 & 16 & 12,5 & 8,5 & 7,5 & 5,5 \\
Densité sèche en place & 1,75 & 1,82 & 2,01 & 2,19 & 2,25 & 2,36 \\
\hline Teneur en eau optimale (\%) & 17,5 & 16 & 15 & 13,5 & 14 & 11,5 \\
Densité sèche maximale & 1,72 & 1,81 & 1,83 & 1,90 & 1,87 & 1,97 \\
Degré de saturation (\%) & 81 & 86 & 83 & 84 & 83 & 81 \\
\hline
\end{tabular}


Après 24 heures d'imbibition, l'échantillon le plus humidifié se transforme pratiquement en pâte et l'échantillon le plus sec est sous forme de grains.

Les résultats moyens des essais de compactage Proctor Normal effectués sont regroupés dans le tableau $V$, en considérant la même division des marnes que précédemment. L'allure des courbes de compactage est symétrique par rapport à l'optimum en général ; la partie humide est pratiquement parallèle à la courbe de saturation.

En ce qui concerne les marnes dures et les marnes intactes, l'optimum Proctor Modifié est de l'ordre de $9 \%$ (teneur en eau optimale) et de 2,1 (densité sèche maximale).

L'indice de consistance à l'optimum PN, Ic $=\frac{\omega_{L}-\omega_{\text {opt }}}{\text { IP }}$ est compris entre 1,1 et 1,3 pour tous les échantillons, sauf avec un échantillon de marne intacte gréseuse où il atteint 1,45. Les matériaux les moins argileux se compactent mieux. La présence de grains assez compacts tend aussi à augmenter la densité (marnes non meubles).

Les marnes meubles ont une teneur en eau naturelle voisine de l'optimum (différence généralement inférieure à 2 points). Les marnes partiellement altérées et les marnes intactes sont du côté sec; l'écart moyen est de 2,5 points pour les marnes tendres (valeurs comprises entre 0,5 et 4,5 ), de 5 points pour les marnes dures et de 6 points pour les marnes intactes. Le rapport de la densité sèche en place sur le maximum PN varie de 1 pour les marnes meubles à 1,2 pour les marnes intactes.

\section{PLANCHE D'ESSAIS}

Afin d'étudier les conditions de compactage des différentes marnes (marnes meubles, marnes tendres, marnes dures, marnes intactes, marnes intactes faiblement et nettement humidifiées), une planche d'essais, implantée près de la butte de Taurine et divisée en six aires d'une longueur de $40 \mathrm{~m}$ et d'une largeur de $4 \mathrm{~m}$, est réalisée en septembre 1979.

Les engins suivants sont utilisés :

- tracteur Caterpillar D9H (puissance de $400 \mathrm{ch}$ ) équipé d'un ripper (dent de $70 \mathrm{~cm}$ ) ;

- décapeuse automotrice Caterpillar 637 (capacité d'une vingtaine de $\mathrm{m}^{3}$ ) ;

- compacteur à pieds dameurs Caterpillar 835 (largeur de $4 \mathrm{~m}$, poids à vide de 30 t et de $35 \mathrm{t}$ avec lest) équipé d'une lame (permettant de niveler les couches);

- un tracteur avec une charrue à socs (Huard, 4 socs en planche) ;

- un camion citerne (muni d'un dispositif d'arrosage).
Les matériaux superficiels (marnes meubles et marnes partiellement altérées) sont prélevés dans les versants de la butte de Taurine entre 0,50 et $2 \mathrm{~m}$ de profondeur. Les marnes intactes sont prélevées entre 3 et $9 \mathrm{~m}$ de profondeur à partir de la crête de la butte de Taurine.

Les marnes partiellement altérées et les marnes intactes déversées sur les aires d'essais par la décapeuse sont essentiellement sous forme d'éléments compris entre $50 \mathrm{~mm}$ et $200 \mathrm{~mm}$ (gros cailloux et blocs). Chaque passe du compacteur 835 est effectuée sur toute la longueur de l'aire; tous les contrôles de densité sont réalisés au milieu (dans le sens de la largeur) de chaque aire et dans les vingt mètres centraux.

Les densités sont déterminées à l'aide d'un densitomètre à membrane et les teneurs en eau à l'aide d'une étuve et d'une balance électriques. Les valeurs données par la suite représentent une moyenne de quatre à huit mesures. L'épaisseur des couches après compactage est de 0,20 à $0,25 \mathrm{~m}$.

Pour les marnes meubles, de teneur en eau égale à l'optímum PN, la densité sèche maximale PN est atteinte avec cinq ou six passes du compacteur 835 . Des passes supplémentaires entraînent un léger matelassage, la densité demeurant constante.

En ce qui concerne les marnes tendres, de teneur en eau égale à $12 \%$, les pieds dameurs parviennent à écraser presque tous les cailloux et blocs de marne. La densité augmente jusqu'à douze passes (à six passes le maximum PN est atteint mais le matériau n'est pas très bien serré), à partir desquelles un léger matelassage apparaît ; le matériau compacté a alors un aspect compact et homogène mais le contour de quelques cailloux de marne peut être observé. Une dizaine de passes du compacteur 835 sont donc suffisantes pour ces marnes tendres de teneur en eau $12 \%$. Pour les marnes les plus tendres (teneur en eau de $16 \%$ ou $17 \%$ ), on peut estimer que six ou sept passes sont suffisantes et pour les moins tendres (teneur en eau de $10 \%$ ) une douzaine de passes.

Le prélèvement des marnes dures $(\omega=8 \%)$ nécessite l'utilisation du ripper du tracteur $\mathrm{D} 9 \mathrm{H}$ pour briser la marne qui est prélevée ensuite par la décapeuse. L'action du compacteur 835 se divise en deux étapes; jusqu'à une douzaine de passes il s'agit essentiellement d'un broyage, d'un concassage du matériau, la densité évoluant peu et le matériau restant assez pulvérulent (la densité sèche mesurée après six passes est égale au maximum PN). Par contre la deuxième étape, entre douze et dix-huit passes, consiste en un serrage efficace du matériau. Après dix-huit passes, la surface compactée est lisse et le creusement de trous montre qu'il n'y a plus de vides visibles, le matériau étant cohérent, très résistant, et ayant un aspect homogène (des essais d'eau confirment son étanchéité) ; on peut toutefois observer le contour des nombreux cailloux de marne encore présents. Bien que les densités sèches atteintes soient alors assez proches du maximum du Proctor Modifié, les comparaisons continuent par la suite à être faites avec l'essai PN. 
Les quatre photos suivantes (fig. $4,5,6$ et 7 ) montrent le prélèvement dans la zone d'emprunt des marnes dures, leur compactage sur l'aire d'essais et l'aspect des trous creusés après six et dix-huit passes du compacteur 835

Les marnes intactes argileuses $(\omega=8 \%$ ), donnent pratiquement les mêmes résultats que les marnes dures. Les tamisages à sec effectués après six passes du compacteur montrent que le matériau est surtout constitué de cailloux et de graviers (il subsiste également quelques blocs).

Quant aux marnes intactes gréseuses $(\omega=6 \%$ ), leur exploitation est encore moins aisée, le ripper s'enfonçant beaucoup plus difficilement. Néanmoins après une vingtaine de passes du compacteur 835 , un bon serrage du matériau est obtenu.

Avec le matériel disponible, l'humidification des marnes intactes argileuses est également expérimentée. Les étapes suivantes sont nécessaires :

- broyage du matériau à Paide du compacteur 835 effectuant six passes;

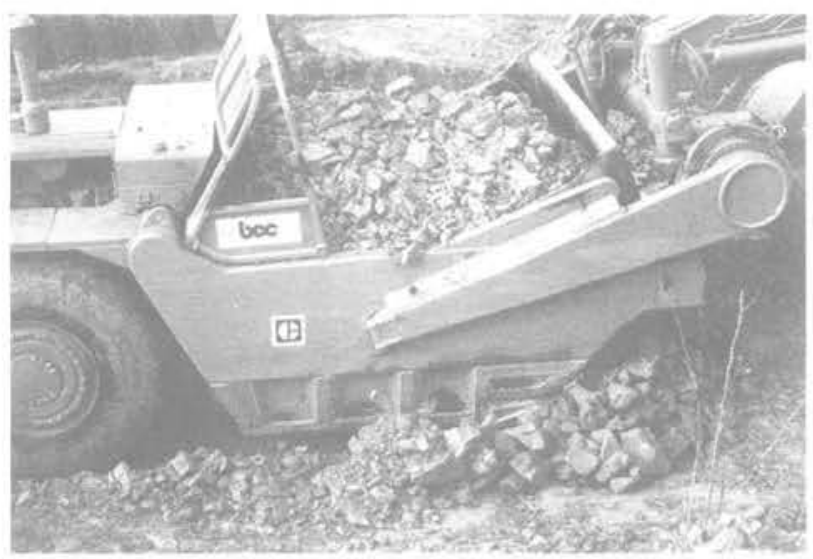

Fig. 4. - Planche d'essais. Prélèvement des marnes dures (partiellement altérées).

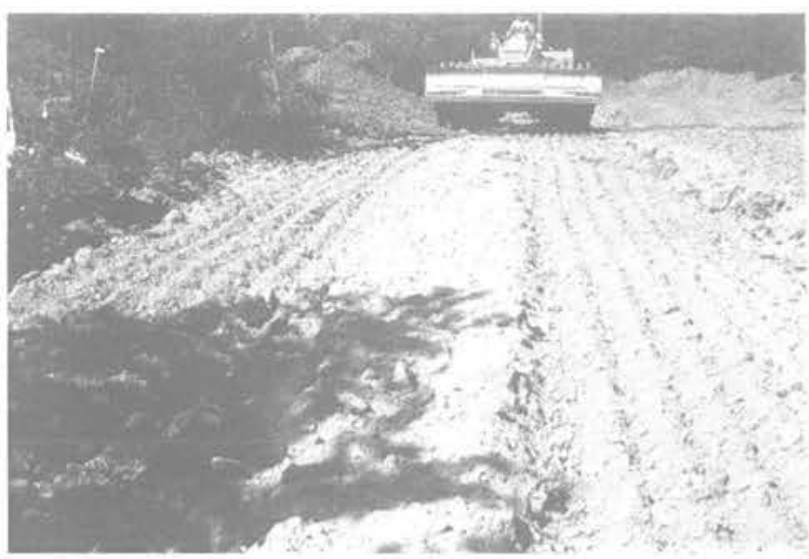

Fig. 5. - Planche d'essais.

Premières passes du compacteur 835 sur les marnes dures (teneur en eau inférieure à $10 \%$ ).
- passage de la charrue à socs pour desserrer le matériau ;

- passage du camion citerne pour arroser la marne (composée d'éléments allant du sable à des blocs) ;

- passage de la charrue à socs pour tenter d'homogénéiser le matériau humidifié; l'opération camion et charrue est répétée trois fois (la teneur en eau augmente en moyenne de trois points)

- pour terminer, une dizaine de passes du compacteur 835 sont nécessaires pour assurer un bon compactage du matériau.

En fait, l'humidification n'est pas du tout homogène : on a une pâte qui enrobe plus ou moins les cailloux présents. L'humidification décrite ci-dessus est donc une solution couteuse et peu satisfaisante.

Sur le tableau VI, les principaux résultats concernant les marnes superficielles compactées à leur teneur en eau naturelle sont regroupés.

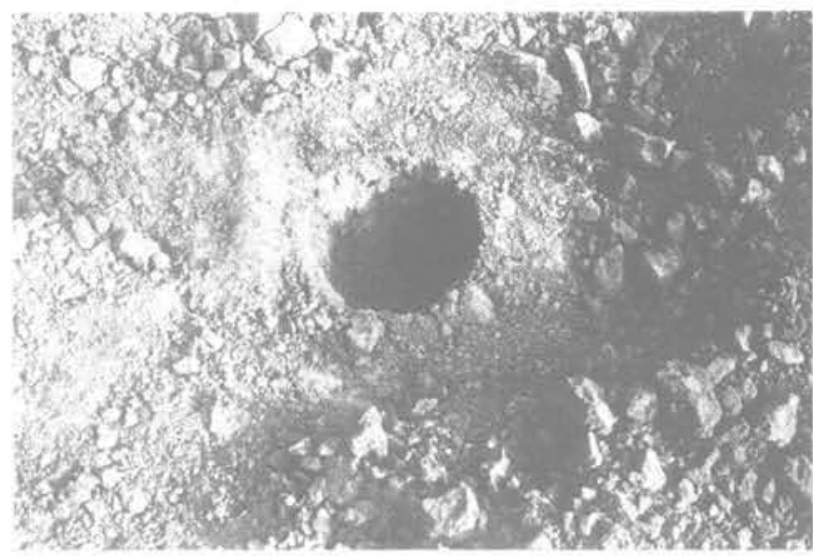

Fig. 6. - Planche d'essais.

Trou creusé dans les marnes dures après six passes du compacteur 835 .

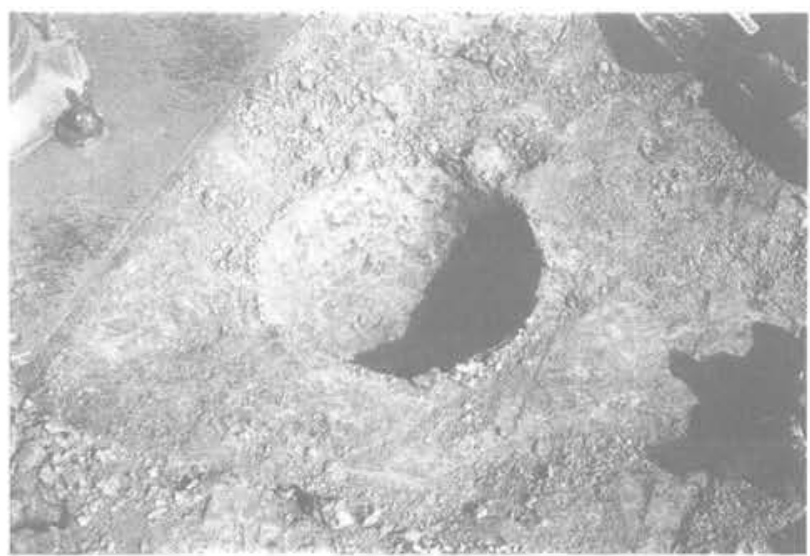

Fig. 7. - Planche d'essais.

Trou creusé dans les marnes dures après dix-huit passes du compacteur 835 . 
A la lecture de ce tableau, on peut donc dire qu'un compactage satisfaisant des marnes de Montbel implique une densité sèche comprise entre la densité maximum $P N$ et la densité en place ; pratiquement la densité satisfaisante est la moyenne de ces deux densités.

Afin de vérifier le comportement des marnes dures peu compactées puis saturées, un échantillon de teneur en eau naturelle de $7,5 \%$ et de densité sèche 2,25 frag. menté en petits cailloux (le tamisage à sec donne $10 \%$ de sable, $30 \%$ de graviers et $60 \%$ de cailloux infé. rieurs à $60 \mathrm{~mm}$ ) est soumis à un essai de compactage à l'énergie PN dans le moule CBR (2,3 litres), ce qui entraîne une fragmentation supplémentaire. Lors de l'essai, la teneur en eau est égale à $4 \%$, le matériau ayant séché. La densité sèche obtenue est de 1,92 valeur probablement voisine du maximum d'un essai Proctor Normal normalisé; comme $\omega_{1}=32 \%$ et IP $=14,5$ on peut en déduire en prenant un indice de consistance à l'optimum PN proche de 1,2 que les valeurs optimales sont $14 \%$ et 1,87 (degré de saturation de $83 \%$ ). Le moule est ensuite plongé, pendant quelques mois, dans un récipient rempli d'eau. A l'ouverture du moule il est constaté que la composition du matériau saturé va d'une pâte assez molle à des cailloux durs. La pâte la plus humide a une teneur en eau de $21 \%$, ce qui correspond à une densité sèche de 1,73 (soit $90 \%$ de 1,92 et $92,5 \%$ de 1,87 ); par contre, les cailloux présents sont dans l'état initial (teneur en eau de 7,5\% et densité sèche de 2,25). Les autres mesures effectuées donnent des teneurs en eau de $10 \%, 15 \%$ et $18 \%$. Cet essai confirme le danger qu'il y aurait à compacter insuffisamment les marnes partiellement altérées et les marnes intactes. Une densité égale à l'optimum PN n'est pas du tout suffisante, le matériau compacté sur chantier étant différent du matériau soumis à un essai $\mathrm{PN}$; en effet au laboratoire, le matériau traité est pratiquement un sol tandis que sur le chantier le matériau est une roche relativement compacte et plus ou moins résistante et déformable, que l'on cherche à broyer et à bien serrer pour éviter la présence de vides (matériau évolutif).

Comme d'une part les essais d'humidification ne sont pas très encourageants et que d'autre part le compactage des marnes dures et des marnes intactes à des densités élevées entraîne des risques de fissuration (matériau sec, hétérogène, peu déformable, sensible au gonflement), il est convenu de n'utiliser pour la construction des remblais que les marnes meubles et les marnes partiellement altérées tendres.

En ce qui concerne le barrage principal (hauteur de $36 \mathrm{~m})$, il est convenu d'utiliser dans la zone amont les marnes meubles et les marnes tendres les moins sèches (teneur en eau supérieure à $13 \%$ ) et dans la zone aval les marnes tendres les plus sèches (teneur en eau comprise entre $10 \%$ et $13 \%$ ), un filtre séparant ces deux zones.

Les pentes du barrage sont peu élevées en raison des caractéristiques mécaniques médiocres des marnes compactées; 1 pour 4 à l'amont et 1 pour 3,5 à l'aval. Le barrage repose sur les marnes non meubles (fig. 8).

Pour la digue de Fajane (hauteur de $20 \mathrm{~m}$ ), il est décidé de réaliser un remblai homogène à l'aide des marnes meubles et des marnes partiellement altérées tendres. Comme les deux parements sont noyés, leur pente est prise égale à 1/4. En fondation, les marnes meubles sont décapées. Côté retenue principale, un dispositif drainant est mis en place (tapis horizontaux). Les études effectuées dans les zones d'emprunt de Fajane montrent que les marnes sont tout à fait similaires à celles proches du barrage principal.

Le chapitre suivant est consacré uniquement au contrôle du compactage de la digue de Fajane, les résultats relatifs au barrage principal ne nous ayant pas été communiqués.

Tableau VI. - Planche d'essais - Résultats

\begin{tabular}{|c|c|c|c|}
\hline Marnes & Meubles & Tendres & Dures \\
\hline $\begin{array}{l}\text { Teneur en eau naturelle }(\%) \\
\text { Densité sèche en place }\end{array}$ & $\begin{array}{c}15,5 \\
1,80\end{array}$ & $\stackrel{12}{2,03}$ & $\begin{array}{l}8 \\
2,22\end{array}$ \\
\hline $\begin{array}{l}\text { Essai Proctor Normal } \\
\omega_{\text {opt }}-\omega_{\text {nat }} \\
\text { Densité sêche maximale } \\
\text { Rapport } \frac{\text { densité en place }}{\text { densité maximale PN }}\end{array}$ & $\begin{array}{l}0 \\
1,80 \\
100 \%\end{array}$ & $\begin{array}{l}2,5 \\
1,85 \\
110 \%\end{array}$ & $\begin{array}{l}5 \\
1,89 \\
117 \%\end{array}$ \\
\hline $\begin{array}{l}\text { Compactage jugé satisfaisant } \\
\text { Densité sèche satisfaisante } \\
\text { Rapport densité satisfaisante } \\
\text { Densité maximale PN } \\
\text { de saturation }(\%)\end{array}$ & $\begin{array}{c}6 \text { passes } \\
1,80 \\
100 \% \\
82\end{array}$ & $\begin{array}{l}10 \text { passes } \\
1,92 \\
104 \% \\
78\end{array}$ & $\begin{array}{l}18 \text { passes } \\
2,06 \\
109 \% \\
67\end{array}$ \\
\hline $\begin{array}{l}\text { Compactage minimal admissible (pour une couche) } \\
\text { Rapport } \frac{\text { densité minimale }}{\text { densité maximale PN }} \\
\text { Degré de saturation }(\%)\end{array}$ & $\begin{array}{c}3 \text { ou } 4 \text { passes } \\
97 \% \\
75\end{array}$ & $\begin{array}{c}7 \text { ou } 8 \text { passes } \\
101 \% \\
71\end{array}$ & $\begin{array}{c}15 \text { ou } 16 \text { passes } \\
106 \% \\
60\end{array}$ \\
\hline
\end{tabular}




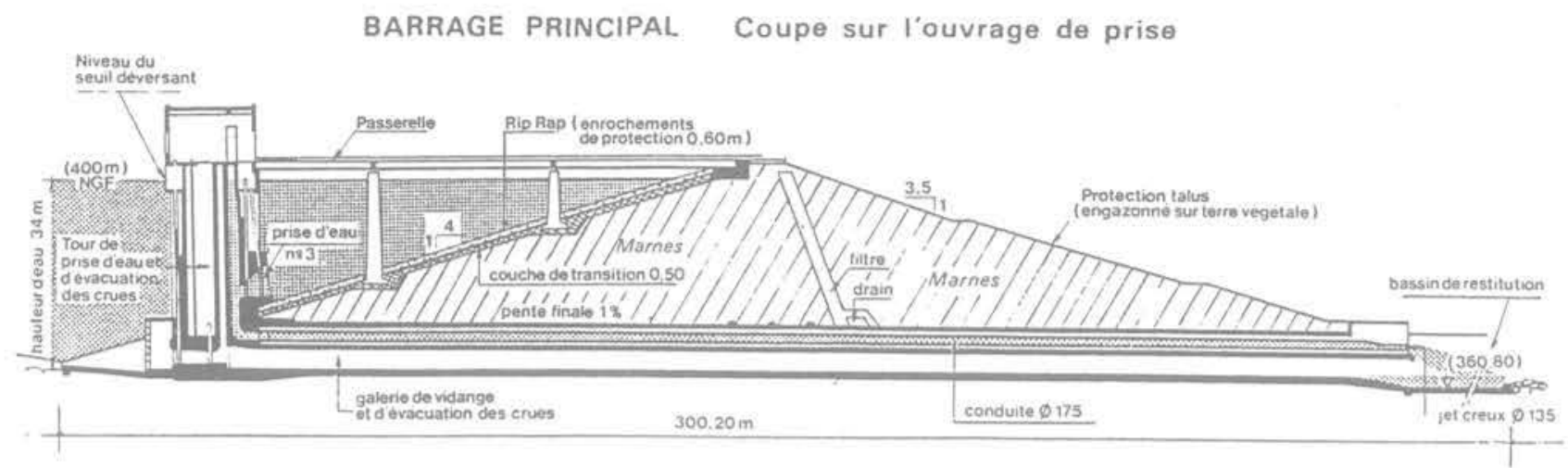

Fig. 8. - Barrage principal.

Coupe sur l'ouvrage de prise.

\section{CONTROLE DU COMPACTAGE DE LA DIGUE DE FAJANE}

Deux rouleaux à pieds dameurs Caterpillar 835 assurent le compactage des marnes. L'épaisseur des couches après compactage est de l'ordre de 0,20 m (avec le matériel disponible, il est possible d'effectuer en

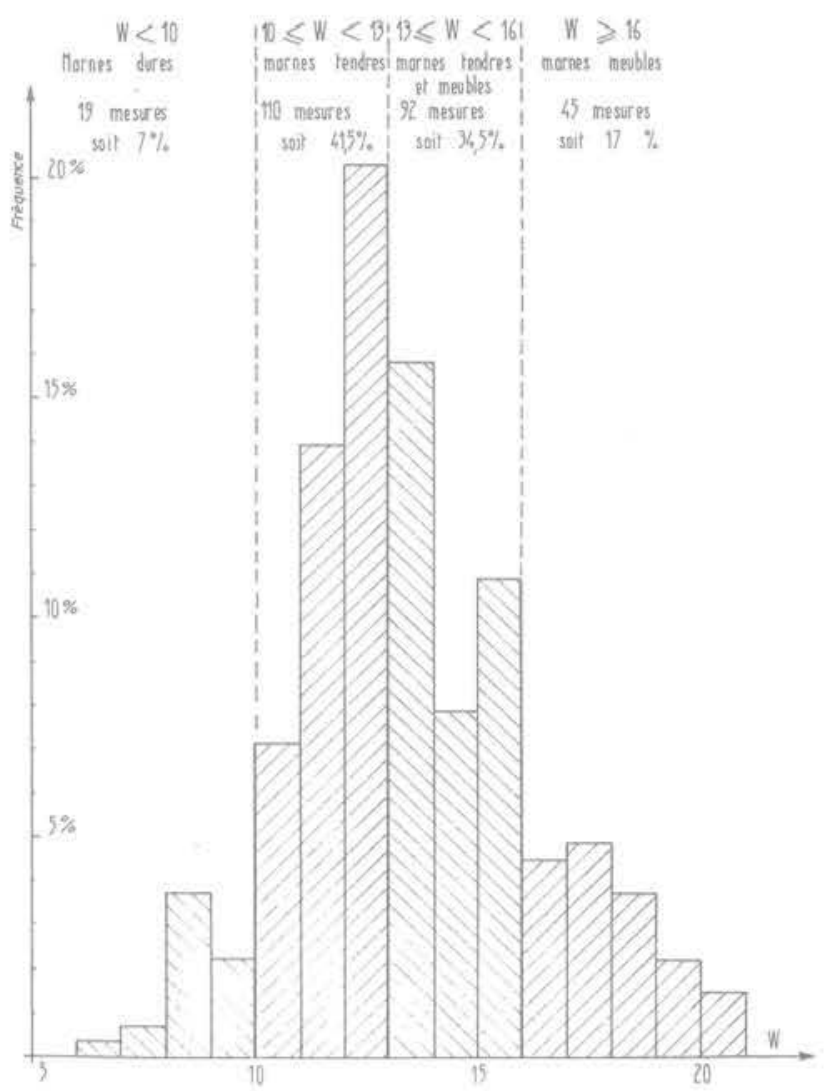

Fig. 9. - Histogramme des deux cent soixante-six mesures de teneur en eau. moyenne une douzaine de passes sur chaque couche). La sonde TROXLER type 3401 est utilisée pour mesurer la densité et la teneur en eau.

L'histogramme ci-après (fig. 9) des deux cent soixantesix mesures de teneur en eau effectuées, montre que le remblai est finalement composé de $7 \%$ de marnes partiellement altérées dures et, en considérant que les matériaux dont la teneur en eau est comprise entre $13 \%$ et $16 \%$ se répartissent également entre marnes tendres et marnes meubles, de $59 \%$ de marnes tendres et de $34 \%$ de marnes meubles.

Comme les matériaux se situent surtout du côté sec par rapport à l'optimum PN et notamment tous ceux susceptibles d'être insuffisamment compactés, le contrôle a été effectué uniquement en fonction du degré de saturation jugé convenable : 60 à $70 \%$ pour les marnes dures, 70 à $80 \%$ pour les marnes tendres.

Le graphe ci-après (fig, 10) montre la répartition des deux cent soixante-six mesures, densité sèche teneur en eau (il s'agit de mesures "finales", après un éventuel compactage supplémentaire). Les trois points concernant le compactage jugé satisfaisant de la planche d'essais de Montbel et les trois points concernant le compactage minimal admissible sont représentés sur ce graphe. Il y a vingt-sept mesures sous la limite admissible mais d'une part certaines en sont très proches et d'autre part les mesures relatives aux marnes meubles ne signifient pas nécessairement que le compactage soit insuffisant.

Trente essais de compactage Proctor Normal ont été réalisés au laboratoire du chantier: la plupart des échantillons ont été compactés peu de temps après leur humidification, ce qui peut avoir eu pour conséquence de décaler légèrement les optimums vers des teneurs en eau plus faibles et des densités plus élevées. Les courbes ont une allure semblable à celle des études du barrage principal; elles sont soit symétriques par rapport à l'optimum, soit légèrement dissymétriques, la partie sèche (gauche) étant alors un peu moins inclinée que la partie humide (pratiquement parallèle à la courbe de saturation). Le degré de saturation moyen des optimums est égal à $84 \%$. 


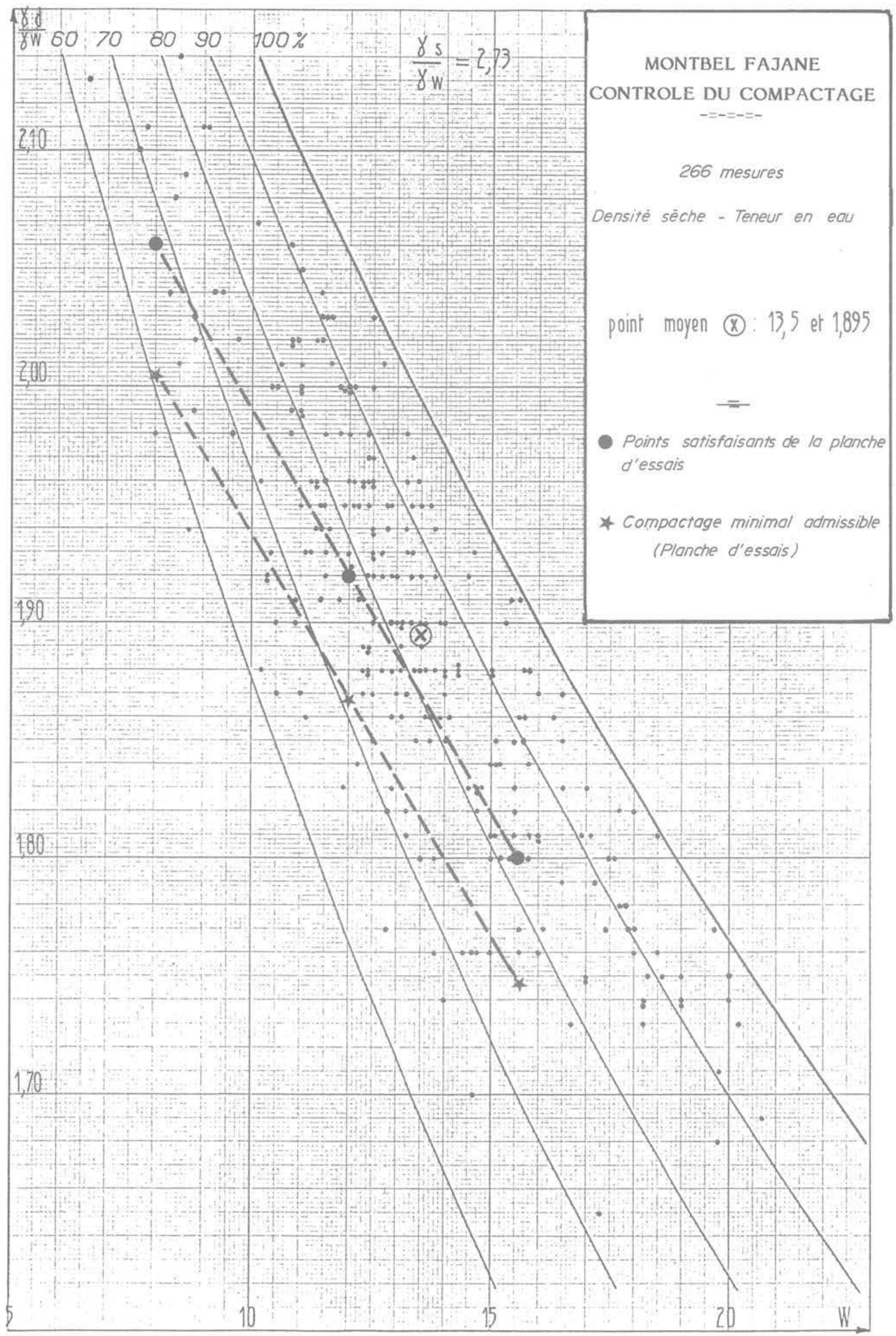

Fig. 10. - Contrôle de compactage. 
La comparaison entre les trente mesures (teneur en eau moyenne de $13,25 \%$ et densité sèche moyenne de 1,90 , valeurs très proches de la moyenne des deux cent soixante-six mesures) et les trente essais PN correspondants (teneur en eau optimale moyenne de $14,75 \%$ et densité sèche maximal moyenne de $1,845)$ montre que globalement le remblai de Fajane a par rapport à l'optimum PN une teneur en eau inférieure de 1,5 points et une densité sèche de $103 \%$.

L'histogramme de la figure 11 donne la répartition des écarts entre la teneur en eau du matériau compacté et la teneur en eau optimale PN. $87 \%$ des matériaux sont situés du côté sec et $13 \%$ du côté humide (il s'agit de marnes meubles).

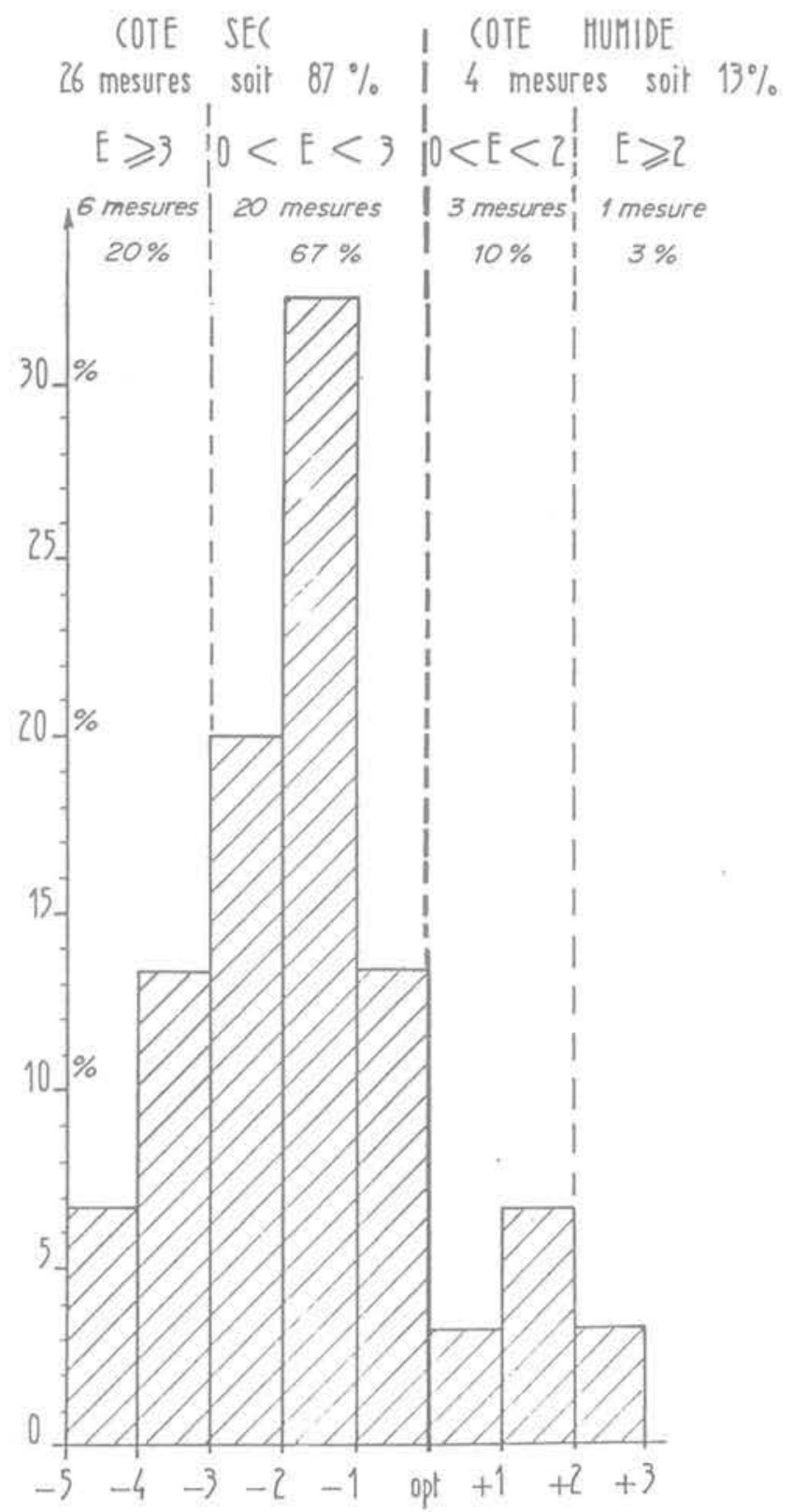

Fig. 11. - Histogramme des écarts $\omega-\omega_{\text {opt }}$ (Proctor Normal) Trente mesures.
Le graphe de la figure 12 permet de comparer chaque mesure de densité sèche et de teneur en eau avec l'optimum Proctor Normal. Du côté humide, les densités obtenues, sauf une, sont inférieures au maximum du PN et du côté sec elles sont généralement supérieures et ceci d'autant plus que le matériau est plus sec.

Du côté humide, on a quatre mesures (marnes meubles) dont la moyenne correspond à un écart de 1,5 points avec la teneur en eau optimale $\mathrm{PN}$ et à un taux de compactage de $97,5 \%$.

Du côté sec, on a vingt-six mesures dont la moyenne correspond à un écart de 2 points avec la teneur en eau optimale PN et à un taux de compactage proche de $104 \%$. Ces vingt-six mesures concernent deux marnes dures, seize marnes tendres dont $\omega<13 \%$ et huit marnes tendres et marnes meubles dont $\omega \geqslant 13 \%$.

Les trois mesures soulignées sont très proches de la droite représentant le compactage minimal admissible (graphe des deux cent soixante-six mesures). La mesure encadrée en est très éloignée mais comme il s'agit d'une marne dont la teneur en eau est égale à $14,6 \%$, cela ne signifie pas nécessairement que le compactage soit très insuffisant.

Avec les seize mesures concernant les marnes tendres dont la teneur en eau est comprise entre $10 \%$ et $13 \%$ on a les résultats moyens suivants :

- à l'optimum PN teneur en eau de $14 \%$ et densité sèche de 1,88 ;

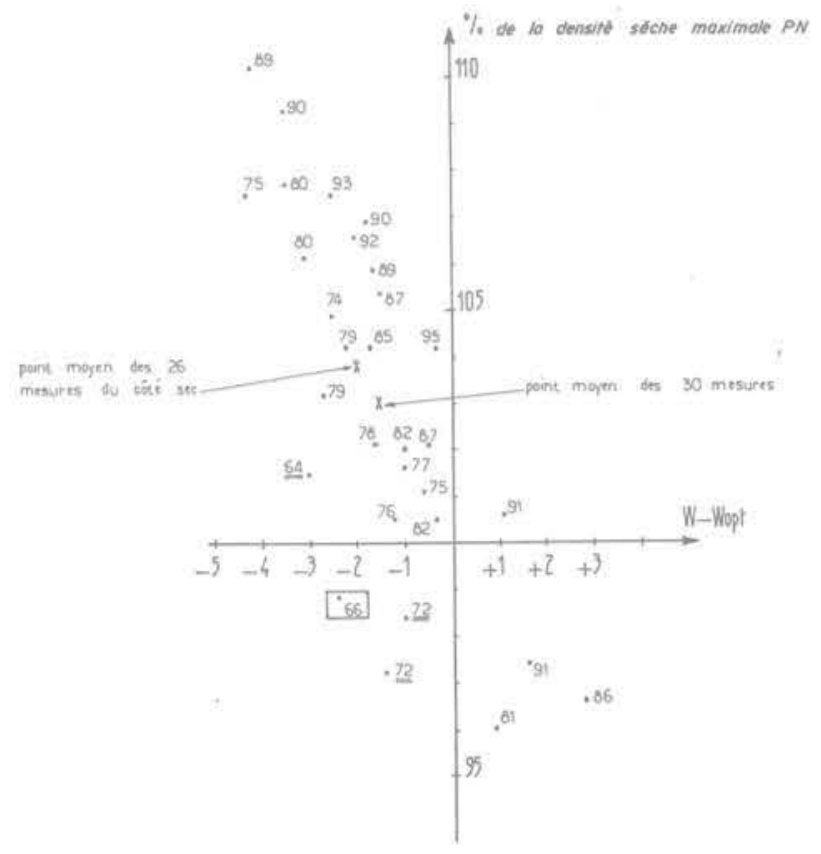

Fig. 12. - Taux de compactage suivant l'écart entre $\omega$ et $\omega_{\text {opt }} P N$ Trente mesures

Chaque mesure est affectée du degré de saturation. 
- teneur en eau naturelle de $12 \%$ (on considère qu'il n'y a aucune évaporation), densité sèche en place de 2,03 et densité sèche mesurée après compactage de 1,95 .

On a donc : $\frac{\text { densité en place }}{\text { densité maximale PN }}=108 \%$ et $\frac{\text { densité mesurée }}{\text { densité maximale PN }}=104 \%$, ce qui est analogue aux résultats de la planche d'essais (densité du matériau compacté entre la densité en place et l'optimum PN).

Si on compare les vingt-six mesures du côté sec avec les optimums PN correspondants en regroupant les mesures en fonction de l'écart $E=\omega_{\text {opt }}-\omega$, on obtient les résultats suivants :

$-E<1$ point : 4 mesures, moyenne $0,4 \%$ et $102 \%$,

$-1 \leqslant E<2: 10$ mesures, moyenne $1,4 \%$ et $102,4 \%$,

$-2 \leqslant E<3: 6$ mesures, moyenne $2,4 \%$ et $104,2 \%$,

$-3 \leqslant E<4$ : 4 mesures, moyenne $3,3 \%$ et $106,1 \%$,

$-4 \leqslant E<5: 2$ mesures, moyenne $4,3 \%$ et $108,8 \%$.

Sur le graphe de la figure 13 on a reporté les cinq points correspondant aux cinq catégories ainsi définies et les résultats de la planche d'essais de Montbel (compactage jugé satisfaisant).

Les mesures effectuées à Fajane concordent tout à fait avec celles de la planche d'essais de Montbel. Il y a proportionnalité entre l'écart $\omega-\omega_{\text {opt }}$ et le taux de compactage.

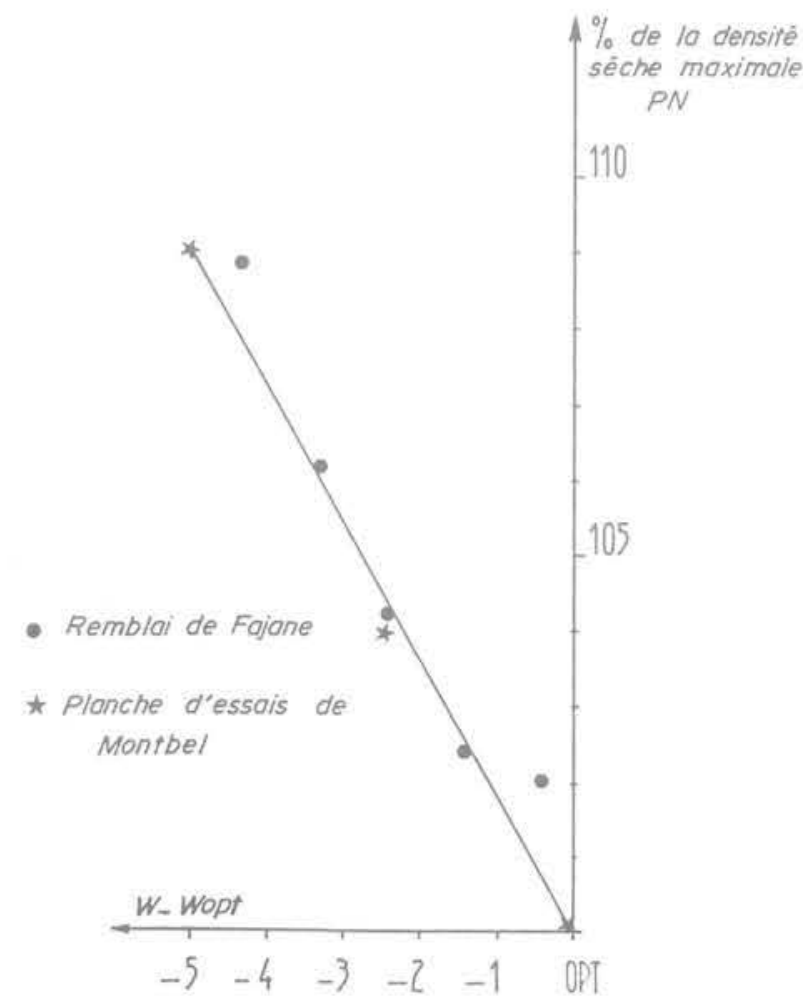

Fig. 13. - Contrôle du compactage.

\section{COMPORTEMENT MÉCANIQUE DU MATÉRIAU SUIVANT LA NATURE DU COMPACTAGE}

$\mathrm{Ce}$ chapitre concerne principalement les essais triaxiaux consolidés non drainés effectués sur des échantillons préalablement soumis d'une part à un compactage en laboratoire (énergie PN, énergie PM) et d'autre part au compactage du chantier (compacteur 835). Des comparaisons sont également faites avec les échantillons non remaniés peu profonds (marnes meubles et marnes tendres) prélevés lors des études des fondations du barrage principal et de la digue de col de hauteur $13 \mathrm{~m}$.

Les essais triaxiaux consolidés non drainés (mesure de la pression interstitielle) ont consisté à écraser à des vitesses lentes, 10 à $20 \mu / \mathrm{mn}$, des éprouvettes de diamètre $35 \mathrm{~mm}$ et de hauteur $80 \mathrm{~mm}$, ces dernières étant préalablement saturées sous une charge d'eau de $2 \mathrm{~m}$ et consolidées sous une contrainte comprise entre 0,05 et $0,4 \mathrm{MPa}$ (ou 0,1 et $0,6 \mathrm{MPa}$ ) pendant une semaine. Il est à noter qu'un échantillon de marne intacte $\left(\omega_{\text {nat }}=8 \%\right.$ et IP $\left.=19\right)$ compacté à l'énergie $\mathrm{PN}$ à une teneur en eau inférieure de 2,5 points à l'optimum a donné les mêmes valeurs de $\varphi$ et c $\left(26^{\circ}\right.$ et $0,01 \mathrm{MPa}$ ) avec les vitesses d'écrasement suivantes : $100 \mu / \mathrm{mn} ; 12 \mu / \mathrm{mn} ; 3 \mu / \mathrm{mn} ; 0,6 \mu / \mathrm{mn}$.

Les résultats des cinquante et un essais effectués (quatre éprouvettes par essai, le plus souvent avec $\sigma_{3}=0,05 ; 0,1 ; 0,2$ et $0,4 \mathrm{MPa}$ ) regroupés en onze séries distinctes, se trouvent dans le tableau VII dans lequel:

- vingt-sept essais concernent les échantillons compactés à l'énergie PN à leur teneur en eau naturelle (sauf les marnes dures et les marnes intactes qui ont été humidifiées); les matériaux ont été prélevés lors des reconnaissances du site, de la réalisation de la planche d'essais et de la construction du barrage principal (il s'agit dans les deux derniers cas de cubes non remaniés d'arête $20 \mathrm{~cm}$ prélevés dans les couches compactées et en partie émiettés)

- treize essais concernent les échantillons non remaniés (cube d'arête $20 \mathrm{~cm}$ ) prélevés dans les couches compactées de la planche d'essais, du barrage principal et de la digue de Fajane,

- six essais concernent les échantillons compactés à l'énergie PM à leur teneur en eau naturelle.

- cinq essais concernent les échantillons non remaniés prélevés dans la zone d'implantation du barrage principal et dans celle de la digue de col de hauteur $13 \mathrm{~m}$.

Tous les essais triaxiaux ont été traités en coordonnées p, q :

$$
p=\frac{\left(\sigma_{1}-u\right)+\left(\sigma_{3}-u\right)}{2},
$$

contrainte principale effective moyenne (centre du cercle de rupture). 
Tableau VII.

\begin{tabular}{|c|c|c|c|c|c|c|c|c|c|}
\hline \multirow{2}{*}{\multicolumn{3}{|c|}{ Échantillons }} & \multicolumn{3}{|c|}{ Identification } & \multicolumn{4}{|c|}{ Triaxiaux consolidés non drainés } \\
\hline & & & \multirow{2}{*}{$\begin{array}{c}\frac{\omega_{\text {nat }}}{\text { en } \%} \\
15,7\end{array}$} & \multirow{2}{*}{\begin{tabular}{|l} 
IP \\
24
\end{tabular}} & \multirow{2}{*}{$\begin{array}{c}\begin{array}{c}\omega_{\text {opt }} \text { PN } \\
\text { en } \%\end{array} \\
16,3\end{array}$} & \multirow{2}{*}{$\begin{array}{l}\varphi \\
24,5^{\circ}\end{array}$} & \multirow{2}{*}{\begin{tabular}{r|}
$\mathrm{c}$ \\
$14 \mathrm{kPa}$
\end{tabular}} & \multirow{2}{*}{\begin{tabular}{|c|}
$\begin{array}{c}\text { Déformation axiale } \\
\text { à la rupture }\end{array}$ \\
12 à $15 \%$ \\
Allure des courbes \\
effort-déformation
\end{tabular}} & \multirow{3}{*}{\begin{tabular}{l}
\multicolumn{1}{|c}{ Remarques } \\
Les cercles sont \\
assez bien «ali- \\
gnés» (même tan- \\
gente pour les \\
4 cercles de rup- \\
ture)
\end{tabular}} \\
\hline \multirow{5}{*}{ 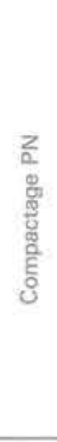 } & \multirow{4}{*}{ 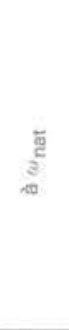 } & $\begin{array}{l}12 \text { échantillons ( } 6 \text { meubles et } 6 \text { ten- } \\
\text { dres). Etude barrage principal }\end{array}$ & & & & & & & \\
\hline & & $\begin{array}{l}4 \text { échantillons (1 meuble et } 3 \text { ten- } \\
\text { dres). Etude digue Fajane }\end{array}$ & 12,7 & 21 & 16 & $24,5^{\circ}$ & $13 \mathrm{kPa}$ & \multirow[b]{4}{*}{$\begin{array}{l}\text { Pratiquement pas } \\
\text { de pic }\end{array}$} & \\
\hline & & $\begin{array}{l}1 \text { échantillon (marne tendre). Plan- } \\
\text { che d'essais }\end{array}$ & 10,4 & 18 & 14,2 & $26,5^{\circ}$ & $16 \mathrm{kPa}$ & & \\
\hline & & $\begin{array}{l}5 \text { échantillons ( } 3 \text { meubles et } 2 \text { ten- } \\
\text { dres). Construction barrage principal }\end{array}$ & 16,1 & 24,5 & 16,4 & $24,5^{\circ}$ & $16 \mathrm{kPa}$ & & \\
\hline & 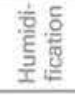 & $\begin{array}{l}5 \text { échantillons ( } 2 \text { dures et } \\
3 \text { intactes). Etude barrage principal }\end{array}$ & 7,3 & 16,5 & 13 & $24,5^{\circ}$ & $22 \mathrm{kPa}$ & & \\
\hline \multirow{3}{*}{\multicolumn{2}{|c|}{ 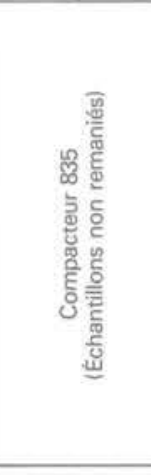 }} & $\begin{array}{l}2 \text { échantillons ( } 1 \text { meuble et } 1 \text { ten- } \\
\text { dre). Planche d'essais }\end{array}$ & 12,2 & 17,5 & 14,1 & $25^{\circ}$ & $30 \mathrm{kPa}$ & \multirow[t]{3}{*}{4 à $14 \%$} & $\begin{array}{l}\text { Pas de diminution } \\
\text { de la résistance } \\
\text { dans le domaine } \\
\text { des fortes con- } \\
\text { traintes }\end{array}$ \\
\hline & & $\begin{array}{l}5 \text { échantillons ( } 3 \text { meubles et } 2 \text { ten- } \\
\text { dres). Construction barrage principal }\end{array}$ & 16,1 & 24,5 & 16,4 & $\begin{array}{r}26^{\circ} \\
\text { Ifail } \\
\text { contra }\end{array}$ & $\begin{array}{l}18 \mathrm{kPa} \\
\text { bles } \\
\text { aintes) }\end{array}$ & & $\begin{array}{l}19,5^{\circ} \text { et } 48 \mathrm{kPa} \\
\text { dans le domaine } \\
\text { des fortes con- } \\
\text { traintes }\end{array}$ \\
\hline & & $\begin{array}{l}6 \text { échantillons ( } 3 \text { meubles et } 3 \text { ten- } \\
\text { dres). Construction digue Fajane }\end{array}$ & 15,7 & 25,5 & 17,7 & \multicolumn{2}{|c|}{$\begin{array}{c}28,5^{\circ} \quad 8 \mathrm{kPa} \\
\text { (faibles } \\
\text { contraintes) }\end{array}$} & & $\begin{array}{l}25^{\circ} \text { et } 18 \mathrm{kPa} \\
\text { dans le domaine } \\
\text { des fortes con- } \\
\text { traintes }\end{array}$ \\
\hline \multirow{2}{*}{\multicolumn{2}{|c|}{ 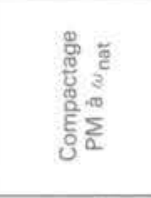 }} & $\begin{array}{l}1 \text { échantillon (marne intacte). Etude } \\
\text { barrage principal }\end{array}$ & 7,5 & 17,5 & 13,3 & $30^{\circ}$ & $35 \mathrm{kPa}$ & \multirow{2}{*}{$\begin{array}{l}8 \text { à } 15 \% \\
\begin{array}{l}\text { Pas de pic pour } \\
\text { deux tiers des } \\
\text { courbes }\end{array}\end{array}$} & \multirow{2}{*}{$\begin{array}{l}\text { Cercles assez } \\
\text { bien "alignés \# } \\
\text { dans l'ensemble }\end{array}$} \\
\hline & & $\begin{array}{l}5 \text { échantillons ( } 3 \text { meubles et } 2 \text { ten- } \\
\text { dres). Construction barrage principal }\end{array}$ & 16,1 & 24,5 & 16,4 & $26^{\circ}$ & $23 \mathrm{kPa}$ & & \\
\hline & & $\begin{array}{l}5 \text { échantillons }(1 \text { meuble et } 4 \text { ten- } \\
\text { dres). Etude barrage principal et } \\
\text { digue de col }\end{array}$ & 14 & 27 & - & $26.5^{\circ}$ & $16 \mathrm{kPa}$ & $\begin{array}{l}4 \text { à } 10 \% \\
\text { (courbes insuffi- } \\
\text { samment prolon- } \\
\text { gées après rupture) }\end{array}$ & $\begin{array}{l}\text { Cercles assez } \\
\text { bien " alignés " }\end{array}$ \\
\hline
\end{tabular}

Tableau VIII.

\begin{tabular}{|l|c|c|}
\hline \multicolumn{1}{|c|}{ Caractéristiques } & $\begin{array}{c}\text { Contrainte de } \\
\text { préconsolidation }\end{array}$ & $\begin{array}{c}\text { Indice de } \\
\text { compression }\end{array}$ \\
\hline Compactage PN à $\omega_{\text {nat }}$ & $0,08 \mathrm{MPa}$ & 0,12 \\
\hline Compactage chantier (échantillons non remaniés) & $0,13 \mathrm{MPa}$ & 0,09 \\
\hline Compactage PM à $\omega_{\text {nat }}$ & $0,12 \mathrm{MPa}$ & 0,11 \\
\hline Fondation (échantillons non remaniés) & $0,12 \mathrm{MPa}$ & 0,10 \\
\hline
\end{tabular}




$$
\mathrm{q}=\frac{\left(\sigma_{1}-\mathrm{u}\right)-\left(\sigma_{3}-\mathrm{u}\right)}{2}=\frac{\sigma_{1}-\sigma_{3}}{2},
$$

contrainte de cisaillement de pic (rayon du cercle de rupture).

On a alors les caractéristiques mécaniques intergranulaires suivantes :

$\varphi=$ Arc sin $(\operatorname{tg} \alpha), \operatorname{tg} \alpha$ étant la pente de la droite de régression obtenue (régression linéaire de q en p),

$\mathrm{c}=\frac{\mathrm{d}}{\cos \varphi}$, d étant le point de rencontre de la droite avec l'axe des $\mathrm{q}$.

Tous ces essais, qui concernent essentiellement les marnes meubles et les marnes tendres, montrent l'influence de la nature du compactage sur le comportement mécanique du matériau.

Le compactage PN et le compactage chantier (compacteur 835) entraînent les différences suivantes:

- dispersion plus importante des valeurs de $\varphi$ et c avec le compactage chantier; dans le domaine des fortes contraintes (contraintes normales effectives supérieures à 0,2 à $0,3 \mathrm{MPa}$ ) une diminution plus ou moins sensible de la résistance est constatée pour neuf essais sur treize :

- déformation à la rupture moins élevée et variable avec le compactage chantier, les courbes effortdéformation $\left(\sigma_{1}-\sigma_{3}\right.$ en fonction de la déformation axiale de l'éprouvette) présentant un pic plus ou moins marqué.

Avec le compactage PM les marnes relativement humides (marnes meubles et marnes tendres) ont des caractéristiques mécaniques légèrement plus élevées (les éprouvettes ont la même densité sèche que les éprouvettes chantier) ; par contre la marne intacte non humidifiée (teneur en eau naturelle voisine de l'optimum PM) atteint une résistance nettement plus forte. Quant aux courbes effort-déformation leur forme est plutôt plus proche de celle obtenue après le compactage PN.

Avec les cinq échantillons non remaniés, prélevés dans les fondations les résultats obtenus sont plutôt semblables à ceux relatifs au compactage chantier.

Les cinq échantillons prélevés dans les couches compactées du barrage principal (trois marnes meubles et deux marnes tendres) ont été soumis à des essais de compressibilité (diamètre de l'éprouvette de $70 \mathrm{~mm}$, hauteur initiale de $24 \mathrm{~mm}$, premier chargement après une semaine de mise en saturation sous $0,60 \mathrm{~m}$ d'eau). Les résultats obtenus sont regroupés dans le tableau VIII en fonction du type de compactage effectué; les résultats concernant les quatre échantillons (une marne meuble et trois marnes tendres) non remaniés prélevés lors des études des fondations figurent également dans ce tableau.

Le compacteur 835 conduit à une plus grande surconsolidation que le compactage PN. La compressibilité des échantillons non remaniés des fondations est assez proche de celle obtenue avec le compacteur 835 .

En fait le comportement mécanique du matériau compacté à l'aide du compacteur 835 est dans l'ensemble plus proche de celui obtenu avec le matériau non remanié prélevé dans les fondations que de celui obtenu avec le matériau préalablement compacté en laboratoire. Le compactage chantier, bien qu'il s'agisse de marnes meubles et de marnes tendres, conserve au matériau la plupart de ses propriétés mécaniques initiales avec toutefois une certaine dispersion dans les résultats obtenus et une tendance à une diminution de la résistance dans le domaine des fortes contraintes.

\section{CONCLUSION}

On a trop souvent tendance d'une part à prendre les mêmes normes de compactage quel que soit le matériau à mettre en place et d'autre part en ce qui concerne les ouvrages relativement importants à utiliser uniquement des compacteurs lourds.

Lorsqu'on a affaire à des matériaux argileux humides (ayant par exemple une teneur en eau supérieure de deux à trois points à l'optimum Proctor Normal), il est préférable pour éviter le développement de pressions interstitielles importantes, de ne pas trop compacter le matériau afin que son degré de saturation ne dépasse pas une valeur de l'ordre de $90 \%$. Une énergie de compactage inférieure à l'énergie PN peut être acceptable. Un engin léger du type rouleau à pieds de mouton est alors certainement plus convenable qu'un compacteur lourd.

Par contre avec des matériaux marneux dont la densité en place est nettement supérieure au maximum du PN, il faut au contraire des engins lourds (du type Caterpillar 835) permettant de bien broyer et de bien serrer le matériau. Il faut obtenir des densités sèches plus élevées que le maximum du PN. A Montbel les essais réalisés ont conduit à préconiser pour le compactage une densité sèche comprise entre le maximum du Proctor Normal et la densité sèche en place. La formule suivante a été établie :

$$
\gamma \mathrm{d} \text { satisfaisante }=\frac{\gamma \mathrm{d}_{\max } \mathrm{PN}+\gamma \mathrm{d}_{\text {en place }}}{2}
$$

Le contrôle du compactage lors de la construction de la digue de Fajane a pu être effectué en considérant le degré de saturation du matériau compacté.

Des différences ont été constatées entre le comportement mécanique des matériaux compactés d'une part sur le chantier et d'autre part en laboratoire. Des études comparatives sont donc nécessaires, notamment pour les grands barrages, le compactage sur le chantier pouvant entraîner un comportement en partie moins favorable.

On peut se demander dans quelle mesure il aurait été possible d'utiliser à Montbel les marnes intactes profondes. D'une part un contrôle très rigoureux du compac- 
tage aurait été indispensable et d'autre part il aurait été nécessaire d'entreprendre une étude très détaillée du comportement du matériau compacté (comportement avec et sans saturation, déformation, gonflement, dessiccation, fissuration), étude difficile à cause de son hétérogénéité (fines et cailloux durs) et de son caractère évolutif.

\section{BIBLIOGRAPHIE}

1. FILLIAT G. et DEPREZ D. - Éléments de géolo. gie, chapitre l de l'ouvrage de FILLIAT G. : La pratique des sols et fondations, Éditions du Moniteur, 1981.
2. HERAUD H., RESTITUITO J., LE ROUX A. Les marnes de Limagne, Association Internationale de Géologie de l'Ingénieur, 3e Congrès, Madrid 1978.

3. LE ROUX A. - Détermination de l'altérabilité des marnes, Association Internationale de Géologie de l'Ingénieur, 3e Congrès, Madrid 1978.

4. ALONSO E. - Remblais et barrages en marne Barrage de Montbel. Thèse $n^{\circ} 389$. Université de Bordeaux 1, 1985. 